\title{
Origin of the magnetic anisotropy induced by stress annealing in Fe-based nanocrystalline alloy
}

\author{
M. Ohnuma ${ }^{\text {a) }}$ and K. Hono \\ National Institute for Materials Science, 1-2-1 Sengen, Tsukuba 305-0047, Japan \\ T. Yanai, M. Nakano, and H. Fukunaga \\ Nagasaki University, Nagasaki 852-8521, Japan \\ Y. Yoshizawa \\ Hitachi Meals Ltd., 5200 Mikajiri, Kumagaya 360-0843, Japan
}

(Received 23 November 2004; accepted 2 March 2005; published online 8 April 2005)

\begin{abstract}
The dependence of the structural anisotropy of Fe-Si-B-Nb-Cu alloy on the applied stress during annealing has been studied by transmission x-ray diffraction. After crystallizing under stress, the Fe-Si nanocrystals show anisotropy in the lattice spacing of the (620) planes. Their elongations are proportional to the applied stress and show a linear correlation with the magnetic anisotropy energy, $K_{u}$. These results indicate that $K_{u}$ originates from a magnetoelastic effect due to an elastic elongation of the Fe-Si phase constrained by the surrounding amorphous phase. (C) 2005 American Institute of Physics. [DOI: 10.1063/1.1901807]
\end{abstract}

Fe-Si-B-Nb-Cu nanocrystalline soft magnetic materials, which are produced by crystallizing melt-spun amorphous ribbons, show soft magnetic properties with a high relative permeability $(>100000)$ and a high saturation magnetization $(>1.0 \mathrm{~T}) .{ }^{1,2}$ In addition, the permeability can be tailored to a specific purpose by inducing a magnetic anisotropy through annealing in a magnetic field ${ }^{2,3}$ or under a stress. ${ }^{4-7}$ The anisotropy energy, $K_{u}$, induced by the latter method, can exceed $5000 \mathrm{~J} / \mathrm{m}^{3}$, a level which is much larger than that induced by annealing in a magnetic field. ${ }^{2-7}$ Because direct observations of the structural anisotropy are lacking, the origin of the stress-induced anisotropy is still under discussion. The following two models have been proposed: (1) a magnetoelastic effect due to the residual stress proposed by Herzer, ${ }^{5}$ and (2) a directional diatomic ordering proposed by Hofmann and Kronmüller. ${ }^{6}$ Varga et al. supported the first model, based on the relation among $K_{u}$ the hardnesses, and the magnetostrictions of a crystalline phase in different $\mathrm{Fe}$ based nanocrystalline alloys. ${ }^{8}$ In contrast, Lachowicz et al. supported the second model because of the temperature dependence of the anisotropy. ${ }^{9}$

The present authors recently found an indication of structural anisotropy in $\mathrm{Fe}_{73.5} \mathrm{Si}_{15.5} \mathrm{~B}_{7} \mathrm{Nb}_{3} \mathrm{Cu}_{1}$ alloy using transmission $\mathrm{x}$-ray diffraction (XRD). ${ }^{10}$ The spacing of the planes of the Fe-Si phase annealed under $200 \mathrm{MPa}$ was elongated in the direction parallel to the tensile stress. Although this result suggested that the stress-induced magnetic anisotropy should be attributed to the magnetoelastic effect, further studies are necessary to establish it. Therefore, to shed light on the origin of the magnetic anisotropy, we have investigated its relationship to the structural anisotropy. This, in turn, demands an understanding of the dependence of the structural anisotropy on the applied tensile stress and its persistence after removal of that stress.

Melt-spun amorphous ribbons with a composition of $\mathrm{Fe}_{73.5} \mathrm{Si}_{15.5} \mathrm{~B}_{7} \mathrm{Nb}_{3} \mathrm{Cu}_{1}$ were heated to $550{ }^{\circ} \mathrm{C}$ at a heating rate of $17.8^{\circ} \mathrm{C} / \mathrm{min}$, maintained at that temperature for $20 \mathrm{~min}$

${ }^{a)}$ Electronic mail: ohnuma.masato@nims.go.jp in an infrared furnace, and then naturally cooled to $100{ }^{\circ} \mathrm{C}$. As a result of heat treatment, the $\mathrm{Fe}-\mathrm{Si} \mathrm{DO}_{3}$ phase (the ordered phase of bcc Fe-Si) is formed in the amorphous phase. A tensile stress was applied to the ribbons during the entire annealing process. The details of the stress annealing process are described elsewhere. ${ }^{7}$ A total of six tensile stresses were loaded: their average values, calculated from the initial sample dimensions, were 10,100,200,300, 400, and $500 \mathrm{MPa}$. During the stress annealing, the ribbons were deformed. Using the final dimensions of the ribbon samples, the corresponding average tensile stresses were estimated to be $10,103,213,334,461$, and $621 \mathrm{MPa}$, respectively. Hereafter, we use the latter values. Some of the samples were reheated to $550{ }^{\circ} \mathrm{C}$ for a certain period of time without applying tensile stress. This reheating causes a relaxation in the magnetic anisotropy of $1-15 \%$.

XRD profiles of these samples were measured in the transmission mode. A monochromated Mo- $K \alpha$ line was aligned to the ribbon with an angle $\theta$ between the incident beam and the normal direction to the sample surface. A transmitted beam with an angle $2 \theta$ from the incident beam was detected. ${ }^{10}$ Consequently, the diffraction vector was fixed to a certain direction in the ribbon plane. We measured the XRD profiles with the diffraction vector parallel to the rolling direction of the ribbon (RD, parallel to the tensile stress), then rotated the sample $90^{\circ}$ and measured the profiles, whose diffraction vector was perpendicular to the RD. To achieve the best compromise between obtaining a stronger intensity (favored at lower angles) as against a larger shift in $2 \theta$ for a certain length unit (favored at higher angles), the peak at about $47^{\circ}$, corresponding to the (620) plane of the ordered Fe-Si phase, was selected for these measurements. All the XRD patterns and sample lengths were measured at room temperature after unloading the tensile stress.

Figure 1(a) shows the magnetization $(M-H)$ curves of the samples annealed under different tensile stresses. The annealing of the ribbons under stress induces a large magnetic anisotropy in the direction parallel to the tensile stress. The XRD profiles of the corresponding samples are shown in 

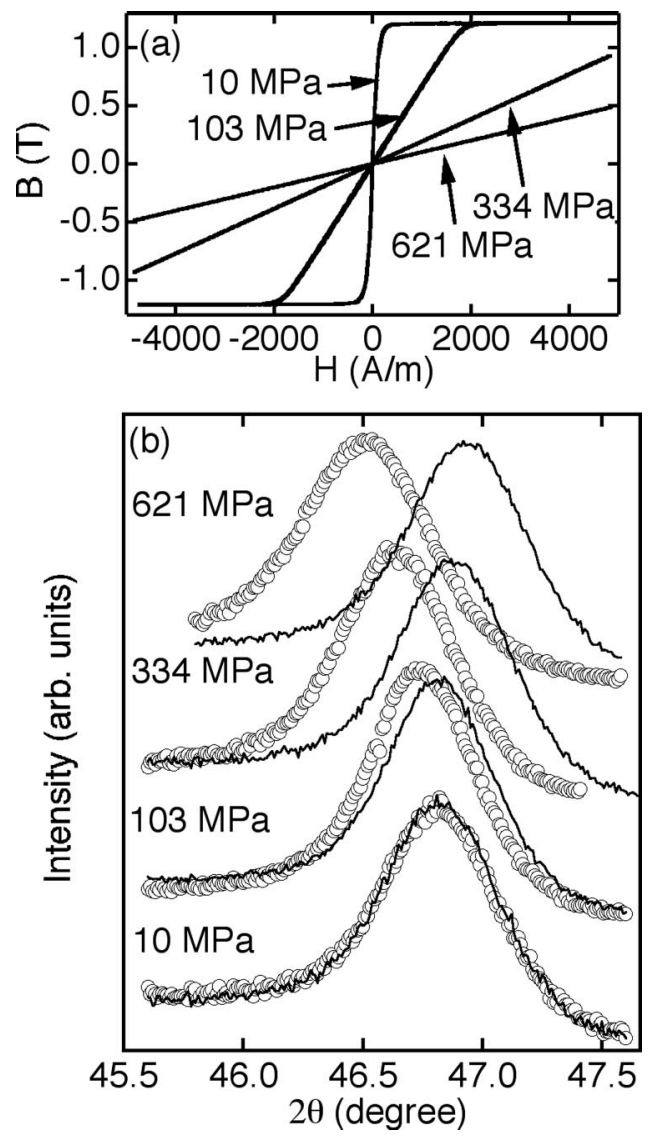

FIG. 1. (a) Magnetization curves and (b) XRD profiles of $\mathrm{Fe}_{73.5} \mathrm{Si}_{15.5} \mathrm{~B}_{7} \mathrm{Nb}_{3} \mathrm{Cu}_{1}$ ribbons annealed under different tensile stresses. All curves in (a) were measured along the RD (parallel to the tensile stress). In (b), the circles indicate a diffraction vector parallel to the RD, while the lines mark a vector perpendicular to the RD.

Fig. 1(b). Increasing the tensile stress induces a large anisotropy of the spacing of the (620) plane. As we previously reported, ${ }^{10}$ selected area electron-diffraction (SAED) patterns of the sample annealed under tensile stress are isotropic, indicating an absence of texture. Therefore, the shift of the peak indicates that the elongation occurs in the direction parallel to the tensile stress. In contrast, a peak shift to a higher angle indicates compression along the perpendicular direction.

Figure 2 shows the changes in the spacings with tensile stress determined from the peak maxima of the curves fitted by a simple Gaussian function. The corresponding strains of the $\mathrm{Fe}-\mathrm{Si}$ phase were calculated as $e_{/ /}=\left(d_{/ /}^{\mathrm{XX}} \mathrm{MPa}\right.$ $\left.-d_{/ /}^{10 \mathrm{MPa}}\right) / d_{/ /}^{10 \mathrm{MPa}}, e_{+}=\left(d_{+}^{\mathrm{XX} \mathrm{MPa} \text { stress }}-d_{+}^{10 \mathrm{MPa}}\right) / d_{+}^{10 \mathrm{MPa}}$, and are indicated on the right vertical axis. The small elongation

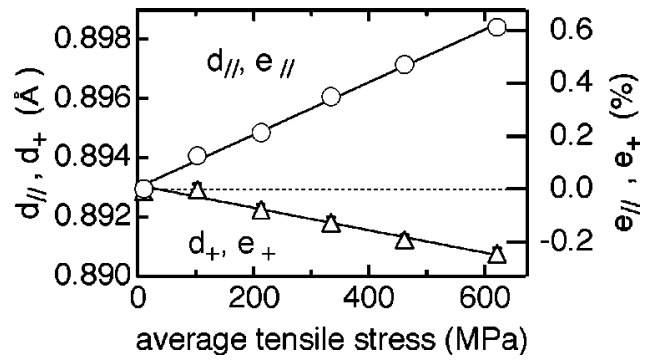

FIG. 2. The spacing of the (620) planes parallel (circles), $d_{/ /}$, and perpendicular (triangles), $d_{+}$, to the RD as functions of the applied tensile stress. Corresponding strains $e_{/ /}$and $e_{+}$are indicated on the right axis.

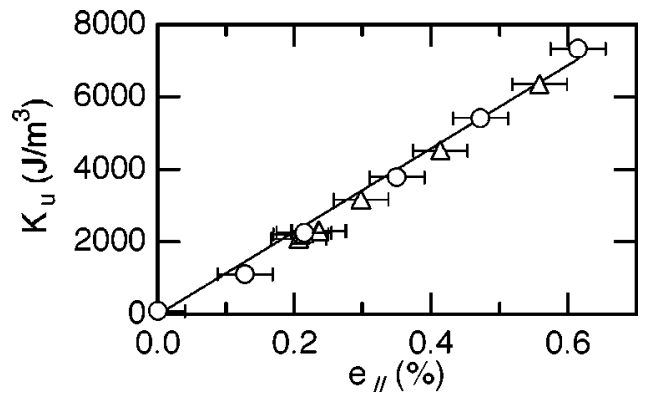

FIG. 3. The anisotropy energy $K_{u}$ as a function of the strain parallel to the $\mathrm{RD}, e_{/ /}$. Circles indicate $K_{u}$ before relaxation, and the triangles after relaxation.

values of less than $1 \%$ observed here were not detected in previous SAED studies. The almost linear relationships seen between the average stress and strains in both perpendicular and parallel to the RD indicate that the observed strains are due to elastic deformations. The Poisson ratio is about 0.37 , a level which is a little higher than the typical value for isotropic porycrystalline metals, $0.3-0.33$. However, because the deviation from isotropic behavior is small, we assume that the deformations determined from the (620) planes are representative of all the $\mathrm{D}_{3}$ planes along the two directions and, for simplicity, treat the material as isotropic in the following analysis.

When an elastic deformation is induced in the magnetic materials, the anisotropy energy $K_{u}$ can be written as $K_{u}$ $=-3 / 2 \lambda_{s} E e_{/ /}\left(\lambda_{s}:\right.$ saturation magnetostriction, $E$ : elastic modulus). ${ }^{11}$ Therefore, the origin of the magnetic anisotropy can be discussed by plotting $K_{u}$ as a function of $e_{/ /}$. As illustrated in Fig. 3, the result is a perfectly linear relation; evidently, the origin of $K_{u}$ is a magnetoelastic effect. The gradient of the fitted line is $1.15 \times 10^{6} \mathrm{~J} / \mathrm{m}^{3}$, i.e., $\lambda_{s} E=-0.77$ $\times 10^{6} \mathrm{~J} / \mathrm{m}^{3}$, a value which agrees reasonably well with the value expected from the Young's modulus ( $\sim 200 \mathrm{GPa})$ (Ref. 12) and the $\lambda_{s}\left(\sim-5 \times 10^{-6}\right)$ (Ref. 5) for polycrystalline Fe-17 at. \% Si alloys $\left(\lambda_{s} E \sim-1.0 \times 10^{6} \mathrm{~J} / \mathrm{m}^{3}\right)$.

When the samples were reheated to $550{ }^{\circ} \mathrm{C}$, a relaxation of the magnetic and structural anisotropies occurred. Figure 4 shows the XRD profiles for the ribbons annealed under $461 \mathrm{MPa}$ with, and without reheating. The magnetic anisotropy energy decreases about $17 \%$ from that of the stressannealed ribbons. After the relaxation annealing, the peak with a diffraction vector parallel to the RD shifts to a higher angle, indicating a lessening of the elongation. As indicated by the triangles in Fig. 3, all the other ribbons, with different stages of relaxation, stay on the same $K_{u}-e_{/ /}$line as before the relaxation. When combined with TEM observations,

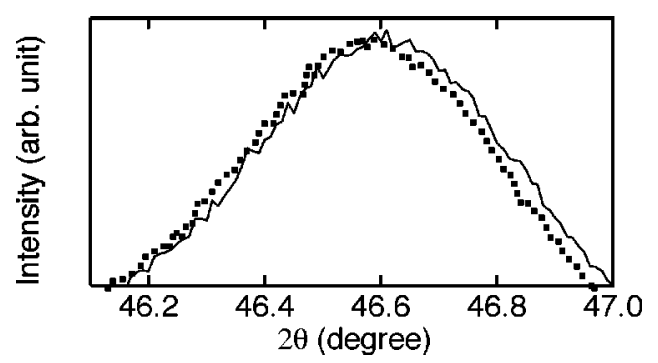

FIG. 4. XRD profiles with a diffraction vector parallel to the RD for the ribbon annealed under $461 \mathrm{MPa}$ (dots) and for the ribbon after relaxation annealing at $550{ }^{\circ} \mathrm{C}$ for $60 \mathrm{~min}$ (solid line).

AIP license or copyright; see http://apl.aip.org/apl/copyright.jsp 


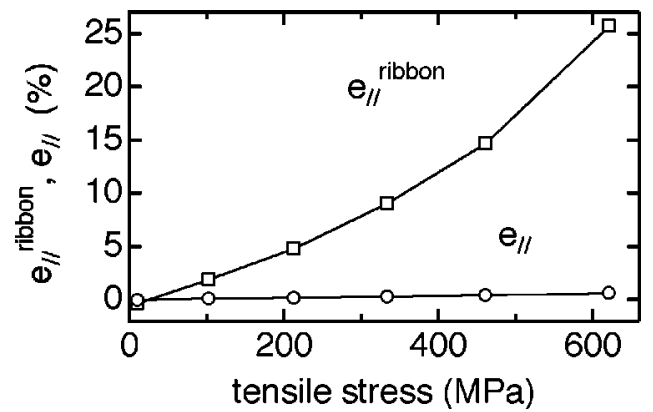

FIG. 5. Elongation parallel to the RD of the ribbon $\left(e_{/ /}^{\text {ribbon }}\right)$ and Fe-Si phase $\left(e_{/ I}\right)$ vs the tensile stress for $\mathrm{Fe}_{73.5} \mathrm{Si}_{15.5} \mathrm{~B}_{7} \mathrm{Nb}_{3} \mathrm{Cu}_{1}$ ribbons.

which show the random orientation and isotropic shape of the $\mathrm{Fe}$-Si phase, ${ }^{4,6,10}$ it can be concluded that the magnetic anisotropy under different stresses is attributable to the magnetoelastic effects of the $\mathrm{Fe}-\mathrm{Si}$ crystalline phase.

In contrast, the above results do not make clear the reason why the structural anisotropy remains after unloading the tensile stress. Figure 5 shows the elongation along the RD of Fe-Si crystals obtained above $\left(e_{/ /}\right)$and that of the ribbons $\left(e_{/ /}^{\text {ribbon }}\right)$ which are determined from the length change of the ribbons as a function of the average tensile stress. Judging from the linear relation of Fig. 2 and isotropic shape of the Fe-Si grains, ${ }^{4,6,10}$ the evidence indicates that no plastic deformation occurs in the crystalline phase. Therefore, the difference between $e_{/ /}^{\text {ribbon }}$ and $e_{/ /}$can be attributed to plastic deformation or viscous flow of the remaining amorphous phase at $550{ }^{\circ} \mathrm{C}$. Since this difference in elongation is already prominent in the ribbon annealed under $103 \mathrm{MPa}$, the yield stress of the amorphous phase must be lower than $100 \mathrm{MPa}$. Assuming a yield stress of $50 \mathrm{MPa}$ and a Young's modulus of $80-160 \mathrm{GPa}$ at $550{ }^{\circ} \mathrm{C}$ (the Young's modulus of the $\mathrm{Fe}-\mathrm{Si}-\mathrm{B}-\mathrm{Nb}-\mathrm{Cu}$ amorphous alloy is $161 \mathrm{GPa}$ at room temperature ${ }^{13}$ ), the elastic elongation of the amorphous phase is in the range $0.03-0.06 \%$ at $550{ }^{\circ} \mathrm{C}$. Therefore, the recovery of the elongation of the $\mathrm{Fe}-\mathrm{Si}$ phase by more than $0.06 \%$ is constrained by the surrounding amorphous phase. Consequently, the elongation of the Fe-Si phase is quenched, even in the stress-free condition.

The elongations of the Fe-Si phase obtained here are about two times larger than the expected value from the Young's modulus of $\mathrm{Fe}-\mathrm{Si}$ at room temperature and the average tensile stress. This discrepancy can be explained by the low Young's modulus of the $\mathrm{Fe}-\mathrm{Si}$ phase at $550{ }^{\circ} \mathrm{C}$, compared to its value at room temperature. In addition, the stress loaded to the Fe-Si phase is higher than the average stress because the remaining amorphous phase is already located in the plastic region.
The contribution of the amorphous phase to the magnetic anisotropy can be roughly evaluated by the equation $K_{u}^{\text {total }}$ where $f^{\mathrm{Fe}-\mathrm{Si}}$, is the volume fraction of the $\mathrm{Fe}-\mathrm{Si}$ crystalline phase, and $\sigma^{\mathrm{Fe}-\mathrm{Si}}, \sigma^{\mathrm{AM}}$, are the residual stress of the $\mathrm{Fe}-\mathrm{Si}$ and amorphous phases, respectively. ${ }^{5}$ Because the amorphous phase has a positive magnetostriction, $\lambda_{s}^{\mathrm{AM}}(+10-20$ $\left.\times 10^{-6}\right),{ }^{5}$ it can add a certain increment to $K_{u}^{\text {total }}$ when under a compressive stress. However, the residual stress of the amorphous phase is complicated, because the stress applied to the amorphous phase is induced by the embedded $\mathrm{Fe}-\mathrm{Si}$ crystalline phase. The amorphous region adjacent to the crystalline phase in the direction perpendicular to the RD is under a compressive stress, while the amorphous part, adjacent to the crystalline phase in the parallel direction to the RD, may be under a tensile stress field. Therefore, the total contribution of the amorphous phase is probably small.

To summarize our study of the structural origin of the stress-induced magnetic anisotropy in $\mathrm{Fe}_{73.5} \mathrm{Si}_{15.5} \mathrm{~B}_{7} \mathrm{Nb}_{3} \mathrm{Cu}_{1}$ alloy by transmission XRD, we have found that the elastic elongation of the Fe-Si phase $\left(e_{/ /}\right)$is quenched in the ribbon. Because the magnetic anisotropy energy $K_{u}$ is proportional to $e_{/ /}$, we have determined that the origin of $K_{u}$ lies in the magnetoelastic effects. The reason for the persistence of $e_{/ /}$is the large difference in the elastic limit of the amorphous phase between the annealing and room temperatures.

This work was supported by the Special Coordination Funds for Promoting Science and Technology on "Nanohetero Metallic Materials" from the Ministry of Education, Sports, Culture, Science, and Technology of Japan.

${ }^{1}$ Y. Yoshizawa, S. Oguma, and K. Yamauchi, J. Appl. Phys. 64, 6044 (1988).

${ }^{2}$ G. Herzer, J. Magn. Magn. Mater. 112, 258 (1992).

${ }^{3}$ Y. Yoshizawa and K. Yamauchi, IEEE Trans. Magn. 25, 3324 (1989).

${ }^{4}$ L. Kraus, K. Zaveta, O. Heczko, P. Duhaj, G. Vlasak, and T. Schnaider, J. Magn. Magn. Mater. 112, 275 (1992).

${ }^{5}$ G. Herzer, IEEE Trans. Magn. 30, 4800 (1994).

${ }^{6}$ B. Hofman and H. Kronmüller, J. Magn. Magn. Mater. 152, 91 (1992).

${ }^{7}$ H. Fukunaga, N. Furukawa, H. Tanaka, and M. Nakano, J. Appl. Phys. 87, 7103 (2000).

${ }^{8}$ L. K. Varga, Zs. Gercsi, Gy. Kovács, A. Kákay, and F. Mazaleyrat, J. Magn. Magn. Mater. 226-230, 1490 (2001).

${ }^{9}$ H. K. Lachowicz, A. Neuweiler, F. Poplawski, and E. Dynowska, J. Magn. Magn. Mater. 173, 287 (1997).

${ }^{10}$ M. Ohnuma, K. Hono, T. Yanai, H. Fukunaga, and Y. Yoshizawa, Appl. Phys. Lett. 83, 2859 (2003).

${ }^{11}$ R. C. O'Handley, Modern Magnetic Materials (Wiley, New York, 2000), p. 235.

${ }^{12}$ T. Yamamoto, The Development of Sendaust and Other Ferromagnetic Alloys (Komiyama, Chiba, 1980), p. 18.

${ }^{13}$ Y. Yoshizawa, Ph.D. thesis, Tohoku University, 1993. 